\title{
Rectal Perforation Associated with a Pelvic Fracture Managed with Lateral Caudal Axial Pattern Flap Surgery Using the Tail to Skin Defect in a Mixed- Breed Dog
}

\section{Jongjin Lee ${ }^{\dagger}$ \\ Jinsu Kang \\ Namsoo Kim \\ Suyoung Heo*}

College of Veterinary Medicine, Jeonbuk National University, Iksan 54596, Korea

Jongjin Lee and Jinsu Kang contributed equally to this work.

*Correspondence: syheo@jbnu.ac.kr

\section{ORCID}

Jongjin Lee:

https://orcid.org/0000-0001-5094-4585 Jinsu Kang:

https://orcid.org/0000-0001-5501-7983

Namsoo Kim:

https://orcid.org/0000-0003-2160-1203

Suyoung Heo:

https://orcid.org/0000-0002-7733-3263

Copyright $\odot$ The Korean Society of Veterinary Clinics
Abstract Complications of pelvic fractures and sacroiliac joint luxation include hemoabdomen, pulmonary trauma, and soft tissue injury. Among them, rectal perforation accounts for $1 \%$ of pelvic fracture complications. Delayed diagnosis is commonly associated with a poor prognosis. A mixed-breed dog was presented to Jeonbuk Animal Medical Center (JAMC) after a traffic accident with no signs of rectal perforation. After fracture surgery, rectal perforation was seen as a perianal fistula. This report describes a case of a perianal fistula progressed from rectal perforation after a traffic accident that was curatively treated with lateral caudal axial pattern flap surgery to the perianal skin.

Key words dog, hindlimb edema, skin flap, burn injury, rectal perforation. 


\section{Introduction}

Pelvic fractures are common in pets, accounting for $16 \%$ of all fractures in dogs and $25 \%$ of all fractures in cats (8). Their main cause is the high prevalence of traffic accidents, and other major causes include falls or bite wounds. The main causes of complications of pelvic fractures involve multiple body systems, such as through pulmonary trauma, hemoabdomen, and soft tissue injury. Rectal perforation accounts for approximately $1 \%$ of pelvic fracture complications $(6,9)$. The prognosis is poor in patients with delayed diagnosis and treatment ( 3 to 7 days after trauma).

This report describes successfully treated case of a rare complication of a pelvic fracture after a car accident.

\section{Case Description}

A 1-year-old, spayed $5 \mathrm{~kg}$ female mixed breed was referred to JAMC after traffic accident. The basic vital signs were stable. The car accident had caused laceration, and the bitch could not walk normally. Neurological examination showed proprioceptive ataxia, which was lost to deep pain. Comput-
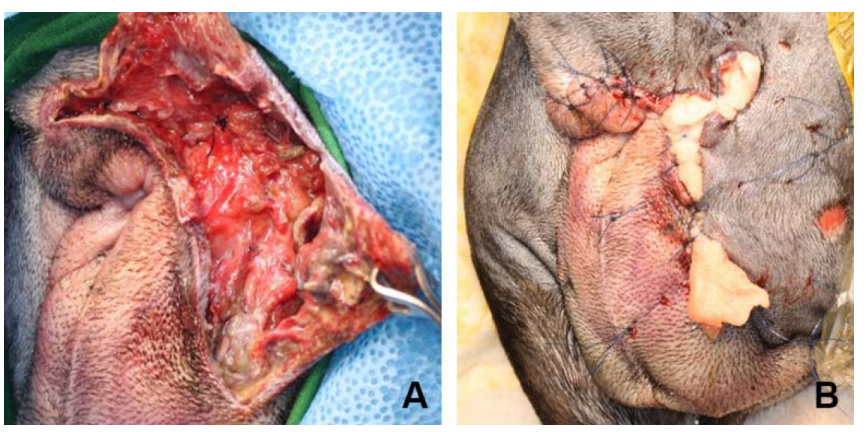

Fig. 1. Rectal perforation repair and debridement surgery (2nd surgery). (A) Sutured perforated rectum in the simple interrupted pattern and the external sphincter and levator ani muscles in the simple interrupted pattern. (B) Defect managed as an open wound because of possible infection. Honey gauze applied to the defect and then lightly sutured. ed tomography and magnetic resonance imaging showed no soft tissue or bone findings and diagnoses of bilateral sacroiliac luxation, right iliac body fracture, bilateral pubic and ischial fractures, and dorsal coccygeal displacement.

Based on diagnostic results, left sacroiliac luxation was operated with a minimally invasive technique using a dilator, and the sacrum was largely fixed using a lag screw (Able Co., Jeonju, Korea). The right side was approached with an open type. Sacroiliac luxation was corrected using a lag screw, and the iliac body fracture was fixed using a 1.5 locking plate (Able Co., Jeonju, Korea). We administered cefazolin (25 mg/ kg, intravenous (IV); Cefozol ${ }^{\circledR}$; Hankook Korus Pharm Co., Seoul, Korea), famotidine $(0.5 \mathrm{mg} / \mathrm{kg}$, intravenous (IV); Gaster $^{\circledR}$; Dong-A Pharm Co., Seoul, Korea), and meloxicam (0.1 $\mathrm{mg} / \mathrm{kg}$, Subcutaneous (SC); Metacam Solution ${ }^{\circledR}$; Boehringer Ingelheim, Ingelheim am Rhein, Germany) as postoperative medication, and tramadol-lidocaine-ketamine combination was provided as constant-rate infusion for pain. Cryotherapy with ice was also performed to reduce overall edema of the limbs.

On postoperative day 4, the overall edema of the limbs had gradually reduced, and the necrotic portion of the skin of the right leg resulting from the traffic accident had been elimi-
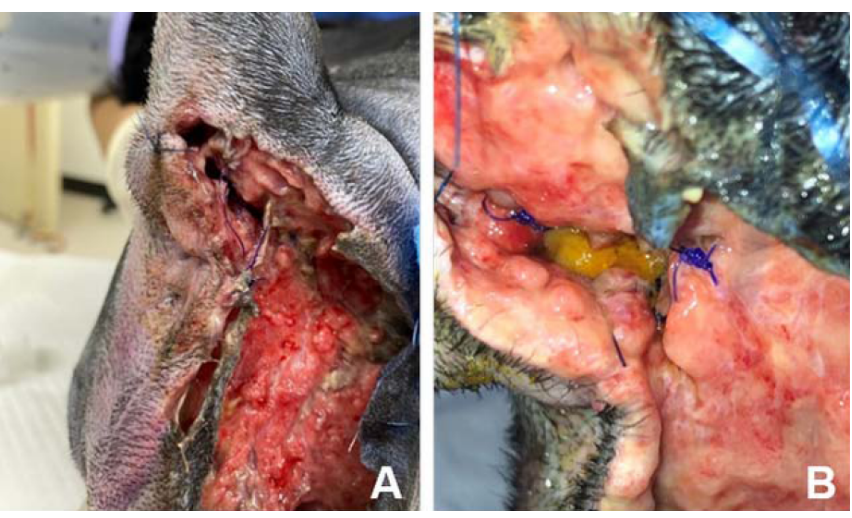

Fig. 2. Open wound appearance after the 2nd surgery. (A) Skin and muscle necroses. (B) Feces leaking from the sutured region again.
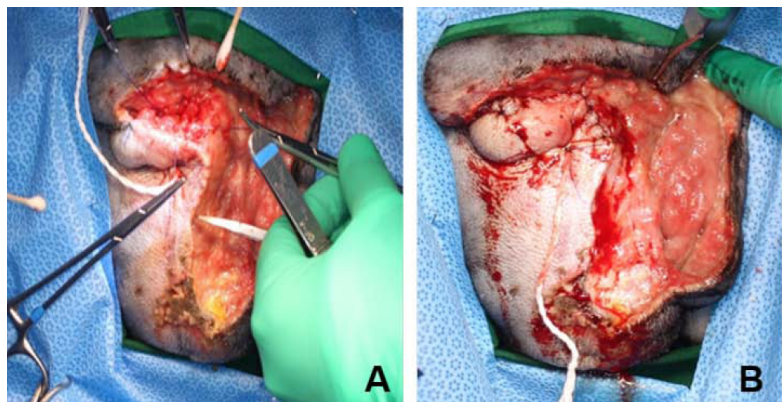

Fig. 3. Rectal perforation repair and debridement surgery (3rd surgery). (A) Perforation sutured with a stay suture. (B) Walking suture applied between the muscles. (C) Gross appearance immediately following surgery. 
nated spontaneously. On postoperative day 7, the C-reactive protein (CRP) had sharply elevated to approximately $150 \mathrm{mg} /$ $\mathrm{L}$ (normal range: 10-20 mg/L). Suturing, present on the right side of the surgical site, was removed, and necrosis was debrided. Since then, the surgical site has been managed as an open wound.

On postoperative day 8, CRP had elevated to approximately $180 \mathrm{mg} / \mathrm{L}$, and the dorsal right portion of the anus was found to have a fistula, containing feces. The rectum had been torn approximately $1 \mathrm{~cm}$ away from the anus.

After an incision of 7-8 cm below the fistula, muscle necrosis and rectal perforation were confirmed, and debridement was performed. The perforated area was sealed with a simple interrupted suture (Fig. 1). After the second surgery, inflammatory indicators, including white blood cell and CRP, reduced in level with healing necrotic skin. However, on postoperative day 4, dehiscence was observed again (Fig. 2). Therefore, we performed surgery with debridement and sutured the rectum (Fig. 3). The central vein catheter was installed to relieve pressure on the intestines and provide total parenteral nutrition. 7 Days after installation, total parenteral nutrition was tolerated well, and CRP remained in the normal range. The central vein catheter was removed on day 7 after the third operation, and nutritional care was changed to canned feed. The postoperative course was uneventful, with no evidence of open wound infection. Therefore, we covered the defect with a skin flap, which was a lateral caudal axial pattern flap obtained from tail skin (Fig. 4).

Postoperatively, mild distal flap necrosis resulted in loss of the ventral margin suture, without additional necrosis or infection. The bitch had no abnormalities of appetite, defecation, urination, proprioception, or deep pain sensitivity.

At the 2-week follow-up after the fourth surgery, the bitch presented with no abnormalities. We stitched the open wound. Walking had improved and recovered to normal at discharge. At the 2-month follow-up after discharge, orthopedic or neurological examination was unremarkable, and hair had grown well in the skin flap (Fig. 5).

\section{Discussion}

Rectal perforation is a rare complication of pelvic fractures and sacroiliac joint luxation. In humans, rectal perforation occurs in $1 \%-2 \%$ of pelvic fractures (2). According to a retrospective study, less than $1 \%$ of 597 dogs with a pelvic fracture were diagnosed with rectal perforation (6). Although rare, delayed diagnosis and treatment can lead to serious complications, including septicemia and disseminated intravascular coagulation. Diagnostic modalities include physical examination, rectal examination, and imaging. In our case, these tests revealed no signs of rectal perforation and, therefore, we ruled out the possibility of rectal perforation. In addition, hemoabdomen, uroabdomen, and thoracic injury, which is commonly caused by trauma, were excluded based on radiography, computed tomography, and vital sign monitoring.

Indicators of rectal perforation for veterinarians can be bloodstaining upon placing a finger in the anus and free
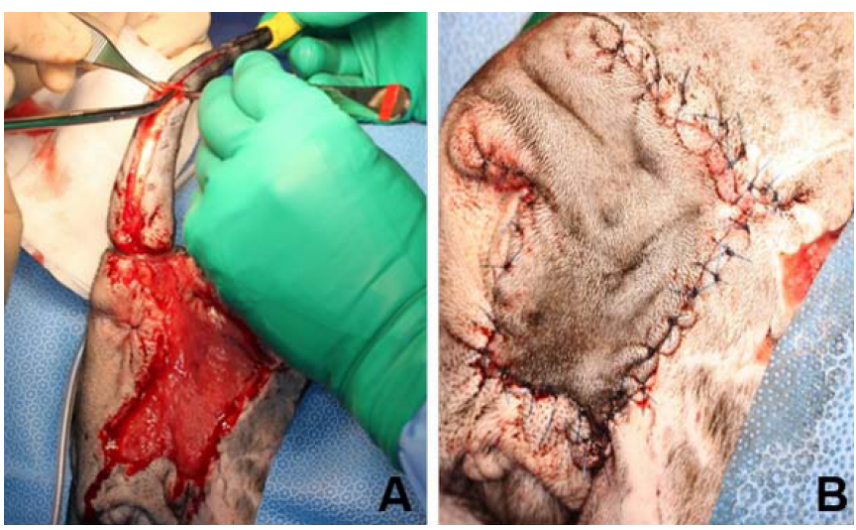

Fig. 4. Lateral caudal axial pattern flap surgery (4th surgery). (A) Fractured tailbone removed from the C2-3 level and skin separated. (B) Postoperative skin flap appearance.
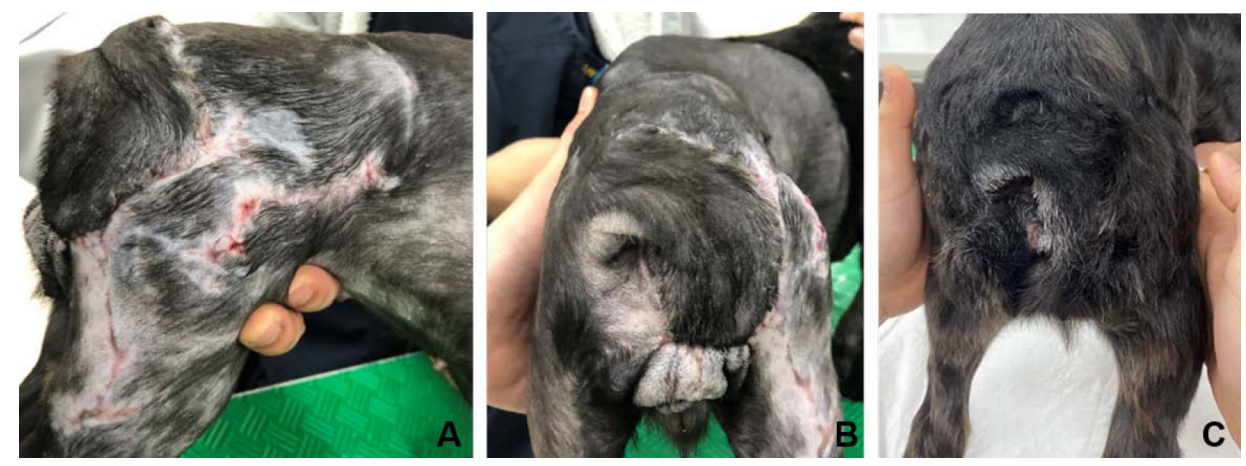

Fig. 5. Skin flap appearance at discharge. (A, B) Skin flap observed to be well-healed without necrosis. (C) Skin flap appearance at two months after discharge. 
gas in the soft tissue of the pelvis. Similarly, in previous reports, electrical perception was suspected through free gas near the workplace, and the treatment was provided early $(1,2,4,5,7,10)$. Computed tomography revealed no signs of free gas in the pelvic region, delaying the diagnosis. A sudden rise in the CRP level and fistula in the trend of decreasing CRP and perianal swelling after pelvic fracture surgery were consistent with rectal perforations on rectal examination.

Accurate physical examination is difficult to perform in early stages, delaying the diagnosis because of severe pain and swelling of the surrounding soft tissue or muscles in rectal perforation (3). These causes can be considered in this case. The patient was probably in a car accident and suffered burns and a fracture. Perforation was also created, but swelling in the area was compressed. Further, feces could not escape, and free gas could not be released. We cannot rule out the possibility of slow rectal perforation as inflammation and necrosis of the surrounding tissues occur and progress. In patients with delayed diagnosis of rectal perforation, the prognosis is poor. However, our patient had a small perforation, underwent immediate surgery, and healed well with the immediate treatment in terms of laboratory changes.

In addition, the patient had extensive burns due to a traffic accident. Further, muscle necrosis progressed because of feces from the fistula, and the skin defect could not undergo primary healing. When observing fresh granulation tissue after open wound care, the patient had a fractured tail, and owner wanted tail resection. Accordingly, we successfully performed lateral caudal axial pattern flap surgery. This case report is limited in that there is only one patient. However, this case may be valuable in the veterinary clinical field owing to the rarity and lack of report of rectal perforation in dogs.

The patient had skin and muscle necroses and rectal perforation, both of which have been successfully cured.

In conclusion, in patients who visit the hospital because of traffic accidents, rectal perforation is rare, but the diagnosis may be delayed. Even if free gas does not appear on imaging, the rectum should be continuously evaluated for reduced swelling because of the possibility of insufficient evidence of perforation due to soft tissue swelling caused by crush damage. Superficial caudal epigastric flap surgery is difficult in cases of a large defect in the perianal region because of frictional heat and a fractured tail bone. In such cases, the lateral caudal axial pattern flap is recommended. In addition, burn injuries are difficult to detect in early cases of black-skinned dogs in a car accident.

\section{Acknowledgements}

This work was supported by the National Research Foundation of Korea (NRF) grant funded by the Korea government (MSIT) (No.2020R1F1A1075219).

\section{Conflicts of Interest}

The authors have no conflicting interests.

\section{References}

1. Clemens MS, Peace KM, Yi F. Rectal trauma: evidence-based practices. Clin Colon Rectal Surg 2018; 31: 17-23.

2. Demetriades D, Karaiskakis M, Toutouzas K, Alo K, Velmahos G, Chan L. Pelvic fractures: epidemiology and predictors of associated abdominal injuries and outcomes. J Am Coll Surg 2002; 195: 1-10.

3. Fransson BA. Rectocutaneous fistulas. Compend Contin Educ Vet 2008; 30: 224-227, 230-235; quiz 235-236.

4. Gwak J, Lee MA, Yu B, Choi KK. Rectal injury associated with pelvic fracture. J Trauma Inj 2016; 29: 201-203.

5. Hoffberg JE, Koenigshof AM, Guiot LP. Retrospective evaluation of concurrent intra-abdominal injuries in dogs with traumatic pelvic fractures: 83 cases (2008-2013). J Vet Emerg Crit Care (San Antonio) 2016; 26: 288-294.

6. Lewis DD, Beale BS, Pechman RD, Ellison GW. Rectal perforations associated with pelvic fractures in sacroiliac fracture-seperations in four dogs. J Am Anim Hosp Assoc 1992; 28: 175-181.

7. Muir P. Rectal perforation associated with pelvic fracture in a cat. Vet Rec 1998; 142: 371-372

8. Phillips IR. A survey of bone fractures in the dog and cat. J Small Anim Pract 1979; 20: 661-674.

9. Streeter EM, Rozanski EA, Laforcade-Buress Ad, Freeman LM, Rush JE. Evaluation of vehicular trauma in dogs: 239 cases (January-December 2001). J Am Vet Med Assoc 2009; 235: 405-408.

10. Tobias KM. Rectal perforation, rectocutaneous fistula formation, and enterocutaneous fistula formation after pelvic trauma in a dog. J Am Vet Med Assoc 1994; 205: 1292-1296. 